\title{
Preference of Diversity Frame When Choosing Universities among Chinese High School Students
}

\author{
Yutong Li' ${ }^{1}$, Dongfang Tian' ${ }^{2 *}$, Yukun $\mathrm{Du}^{3^{*}}$ \\ ${ }^{1}$ Peking University, Beijing, China \\ ${ }^{2}$ Beijing No. 80 High School, Beijing, China \\ ${ }^{3}$ Sun Yat-sen University, Guangzhou, China \\ Email:1907096379@qq.com
}

How to cite this paper: Li, Y. T., Tian, D. F., \& Du, Y. K. (2020). Preference of Diversity Frame When Choosing Universities among Chinese High School Students. Psychology, $11,445-460$.

https://doi.org/10.4236/psych.2020.113030

Received: December 14, 2019

Accepted: March 15, 2020

Published: March 18, 2020

Copyright () 2020 by author(s) and Scientific Research Publishing Inc. This work is licensed under the Creative Commons Attribution-NonCommercial International License (CC BY-NC 4.0).

http://creativecommons.org/licenses/by-nc/4.0/

\begin{abstract}
This study seeks to examine the relationship between Chinese high school students' schooling abroad experience and their preference for instrumental or moral diversity frame, and further explore the mediation mechanism. In the present study, we take schooling abroad experience as the independent variable, and take attending preference of university with moral or instrumental diversity frame as the dependent variable. We have two sets of mediators: the first set of mediators are perceived minority/majority based on past experience and severity of unfair/biased experience based on nationality, and the second set of mediators are expected value, sense of belonging and possibility of being treated with bias in two universities. We send out questionnaires to 100 Chinese high school students. The statistical methods used in our analysis were ANOVA, correlation, and regression. The results are partly contrary to our expectation as there was no significant relationship between schooling abroad experience and preference of diversity frames, as well as severity of unfair/biased experience. The only validated path is between perceived minority/majority and preference of diversity frames, which is mediated by participants' expected sense of belonging and possibility of being treated with bias at the instrumentally motivated university. Then, we explain our results considering status of Asian Americans in the United States, adjustment and some other aspects.
\end{abstract}

\section{Keywords}

Diversity Frame, Chinese High School Students, Minority, Majority, Unfair Experience

*These authors (listed according to alphabetical order of the first name) contributed to this work equally. 


\section{Introduction}

With increasing globalization, the concept of diversity has been gradually popularized worldwide. Especially in countries like the United States where equality and inclusion are strongly emphasized, diversity is highly valued by both business organizations and higher education institutes. As far as universities are concerned, diversity can prepare their students for the challenge of globalization, and also can keep them vigorous and competent in academic area. A representative of international students, Alice Zurek, pointed out that she enjoys meeting students from various backgrounds, which further showed that diverse environment which overseas schools provide resembles a melting pot where interracial interactions are encouraged with people from multicultural backgrounds (Nabi, 2016). Beyond this, without the inherent racial limitations, benefits of framing diversity in educational context reach far across a range of areas, including better academic achievements-students' higher test scores and graduation rate-and mental health since there is less pressure brought by reduced racial bias and stereotypes (Hoxworth, 2018). For these reasons, almost all universities in the United States have a department to maintain and promote diversity and equality on campus. Thus, the effects of diversity and how to promote it in the context of higher education have attracted wide attention of psychologists. For social psychologists, making sure that the diversity or inclusion policy can bring tangible benefits to students (especially minorities) is an important issue on which they focus. For example, there are abundant researches proving that diversity can positively influence peoples' cognitive skills (e.g. critical thinking and problem-solving skills), increase cultural awareness and higher levels of civic engagement (Trawalter, Driskell, \& Davidson, 2015).

However, extant studies about diversity seldom explored the reason why we should encourage diversity. Based on previous studies, there are two types of arguments people usually propose when explaining why we have to embrace and promote diversity-moral diversity frame and instrumental diversity frame. The moral diversity frame stresses the equality and moral value of diversity, implying that creating a more diverse organization is a valued goal in and of itself (i.e. diversity is fair). In contrast, the instrumental diversity frame emphasizes the beneficial outcomes, which diversity can bring to the organization. In this case, diversity is only a means to some other end (i.e. diversity is good).

Though currently in America, equality is what the whole society emphasizes and has historical significance, instrumental diversity frame is actually more prevalent according to previous studies (Trawalter et al., 2015; Starck, Sinclair, \& Shelton, 2019b). The prevalence of instrumental diversity frame is quite reasonable as we are embracing a globalized society and instrumental diversity frame can indeed bring various positive effects to the organization and not only Black Americans but also White Americans are aware of how they can benefit from diversity. Nevertheless, it has raised questions about whether the instrumental diversity frame can bring equal rights to racial majorities and minorities. Tra- 
walter et al. (2015) has found that the instrumental diversity frame has costs as using it could broaden the definition of "diversity", deprioritize the blacks while recruiting, decrease the likelihood of selecting a more devalued group and encourage whites to consider diversity in the university as a compromise without understanding its moral value and lose motivation to embrace diversity. However, the moral diversity frame has costs, too. For instance, making diversity as an uncompromising and direct standard in an organization may let White Americans feel excluded and also worry too much about being considered as prejudiced or discriminated (Vorauer, Hunter, Main, \& Roy, 2000). This kind of social threat could stop White Americans from interacting with Black Americans or other racial minorities, rather than fuel those interracial contacts.

In this case, it is natural that different ethnic groups have different preferences toward diversity frames, as they may get unequal benefits from them. Starck et al. (2019a) studied the rationale of people's preference toward moral and instrumental diversity, and found that White Americans prefer instrumental diversity rationales over moral ones. In contrast, they found that minorities display a completely different set of preferences from Whites-minorities in general preferred a moral diversity rationale over an instrumental one. Here minorities refer to Black, LatinX and Asian Americans. However, there are few extant literatures which have studied non-white international students' preferences for moral and instrumental diversity rationales. Among all of the non-white international students, Chinese students take up a large part and now form the largest proportion of international students (31\%), with undergraduate enrollment increasing more than eightfold in just six years (Heng, 2018). So, we choose Chinese high school students as our main focus and we intend to investigate their preference for moral and instrumental diversity frames while choosing universities in the current study. As there are limited literatures for us to infer Chinese high school students' preference directly, it is reasonable to form the hypothesis based on following aspects.

First of all, Chinese high school students will become minorities in American universities, so their preference might be similar to racial minorities in the USA (e.g. Blacks, LatinXs), who prefer the morally motivated university. However, when looking further into this inference, it is obvious that Chinese high school students should not be regarded as a whole because they have had different experiences with regard to minority or majority status, racial bias or concept of diversity. One possible explanation for these differences may be their schooling experience. For those who have never been studied abroad, they have always been studying and living in an environment with little racial bias, and paying less attention to equality or diversity. But for those who have studied abroad, they have had experience of being a minority or living in a culture that strongly values equality and diversity. As a result, we take Chinese high school students' schooling abroad experience as the independent variable and we want to test whether it is related to their preferences for moral and instrumental diversity frames. 
Then we hope to further test the mechanism through which the schooling abroad experience influences Chinese high school students' preferences for different diversity frames. According to previous studies, discrimination is one of the most common and salient stressors that Asian international students experience and the discrimination they report is at higher rates than that is reported by their European counterparts (Wong, Tsai, Liu, Zhu, \& Wei, 2014). This situation is identical to what Black Americans are dealing with in their daily life. As a stigmatized group, Black Americans are easy to be stereotyped, negatively evaluated, or discriminated against on the basis of their race (Starck, Sinclair, \& Shelton, 2019a). Similarly, stereotypes based on Asian international students' nationality and race are also major causes of their experiences of discrimination. Thus, we hypothesize that unfair or biased experience based on nationality is a mediator of the relationship between schooling abroad experience and participants' preference for diversity frames.

While unfair or biased experience related to nationality is an external factor which may play a role in both Black Americans and Chinese high school students' forming their preference for different diversity frames, we propose an internal factor-perceived minority/majority, which may also play an important part in the formation of preference for diversity frames. Perceived minority/majority status is an internal factor which has similar but not the same meaning to racial minority/majority status, as it also contains the process of subjective interpretation. Starck and Sinclair (2019) found in their studies that racial identification can moderate whether Black Americans prefer moral diversity frame. In this case, racial identification is an internal factor that related to one's subjective perceptions in addition to their inborn racial identity. When perceiving themselves more as minority rather than majority, Black Americans may get a higher racial identification, more willingness to learn about related discriminated history, etc., and that can lead to a more positive attitude toward moral diversity frame in the end.

After proposing two possible mediators between the independent variable (i.e. schooling experience) and the dependent variable (i.e. preference for moral and instrumental diversity frames), we'd like to further investigate the mechanisms from which participants' unfair/biased experience and perceived minority/majority may influence their final preference for different diversity frames. Starck et al. (2019a) proved that at least three factors contribute to divergence between White Americans' and Black Americans' preference for diversity frames. As for White Americans, they expect to benefit more, experience less social identity threat and feel more included in instrumentally motivated universities. In contrast, minorities in general preferred a moral diversity frame over an instrumental one, because minority students expect to benefit more, belong more, and experience less social identity threat at a morally motivated university (Starck, Sinclair, \& Shelton, 2019b).

In the present study, we proposed a mediating model to examine the relationships among Chinese high school students' schooling abroad experience, un- 
fair/biased experience, perceived minority/majority and three mediators (i.e. participants' expected value, sense of belonging and possibility of being treated with bias) (see Figure 1). This study set out to address gaps in the existing literature in international students' preference for moral and instrumental diversity frames, and further explore the mediating mechanisms. The study investigated which diversity frame Chinese high school students preferred when choosing the university. To further operationalize "schooling abroad experience" (i.e. the independent variable), we measure the length of their abroad experience and anticipate that it will influence Chinese high school students' preferences for different diversity frames, as the longer time they study abroad, the better they can adjust to or integrate into their surroundings, so they might not prefer moral diversity frame as much as those who has only studied abroad for a short period do.

The aims of the present study were threefold. First, we aimed to investigate the relationship between schooling abroad experience (never/short-term/long-term) and preference for diversity frames (moral/instrumental). Specifically, we hypothesized that those who had short-term schooling abroad experience would prefer moral diversity frame the most, then the students who had long-term schooling abroad experience, and those who have never studied abroad would prefer instrumental diversity frame rather than the moral one.

Second, we aimed to investigate whether unfair/biased experience and perceived minority/majority could mediate between schooling abroad experience and the preference for different diversity frame. To be exact, we wanted to test the hypothesis that schooling abroad experience would lead to more unfair/biased experience and the tendency to perceive themselves as minority rather than majority first, and then test whether these two mediators correlate with participants' preference for different diversity frames.

Third, we tested the hypothesis that expected value, sense of belonging and possibility of being treated with bias would mediate between unfair/biased experiences, perceived minority/majority and preference for instrumental or moral diversity frame.

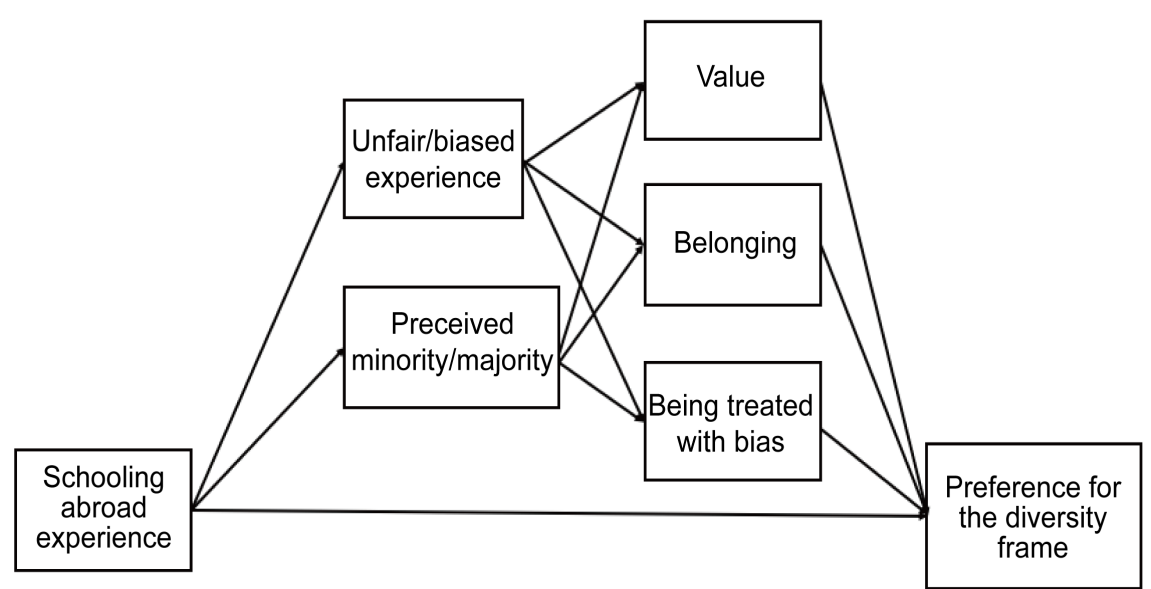

Figure 1. Mediation model predicting the preference for the diversity frame. 


\section{Method and Materials}

\subsection{Participants}

108 Chinese participants were recruited through convenience sampling both online and offline, who were high school students intended for schooling abroad for university and were willing to fill out our survey. We excluded 6 participants, 2 of whom showed evidence of responding very carelessly, and 4 of them were undergraduate students (we only wanted high school students). Our final sample included 102 participants ( 51 male, 51 female) from 15 - 19 years of age ( $M=16.94$, $S D=0.80)$. We categorized the participants based on whether they have experience of schooling abroad or not, and those who have experience abroad were further divided into two groups based on the length of time, with $50 \%$ of them in the short term abroad group and another $50 \%$ in the long term abroad group. So the final divisions included three groups: never abroad $(n=51)$, short-term abroad $(n=25)$, and long-term abroad $(n=26)$.

\subsection{Procedure \& Materials}

Participants were asked to scan our QR code and complete the questionnaire online. In the questionnaire, they had to answer 22 questions in total. The questionnaire included three sections. Unless otherwise indicated, all the items in were on 7-point Likert scales. The setting and order of the questions in our questionnaire are based on the mediation model in our hypothesis. The questions in every part correspond to the corresponding variables in the intermediary model that we want to explore.

Section 1 had 15 questions. We asked about their gender, age, whether they were in high school or not, the location of their school and the length of their abroad schooling experience. The compilation of the basic information about schooling experience is based on the information learned in previous interviews. We learned from the interview that the high school students' schooling is basically divided into long-term overseas education, short-term overseas exchange or study tour and other experiences, and the type and time of studying abroad may lead to different feelings. Then there were three items we set as distractors, hoping to prevent participants from knowing the exact purpose of this questionnaire. These three items were about their school life, for example "How much do you enjoy going to your current school?".

Then we asked participants to rate the degree to which they felt like a member of the minority versus the majority based on their past schooling experience. "How much do you think you belong to minority/majority based on your past schooling experience?" and they were asked to rate on Likert scales with 1 as "Minority" and 7 as "Majority". This question is designed based on "perceived minority/majority" in the mediation model and last two questions were about their unfair or biased experience based on nationality "Have you ever been treated unfairly or with bias based on nationality in your past experience?", which cor- 
responding to "unfair/bias experience" in the model. For this question, participants choose "yes" or "no" and if the answer is "no", they do not need to answer the next question. The next question is "Please rate the degree of severity" and they were asked to rate on Likert scales with 1 as "Very fair/unbiased" and 7 as "Very unfair/biased".

In Section 2, participants were asked to read statements describing two universities with different diversity frames (i.e. instrumental diversity frame and moral diversity frame). These statements were first used in an extant paper (Starck, Sinclair, \& Shelton, 2019a) as instrumental and moral diversity frame statements. We modified the moral one and added some words to strengthen its emphasis on equality.

The moral statement read:

"For us, diversity is about justice and ensuring that every single person from different backgrounds has equal opportunity to receive excellent education. It will be a priority for our university." - statement from the President.

The instrumental statement read:

"For us, diversity is about enriching students' intellectual experience and preparing them to success. It will be a priority for our university."-statement from the President.

The order in which the diversity frames were presented was counterbalanced across participants, which means that for half of the participants, University A was associated with the instrumental statement and University B was associated with the moral statement, and for another half the statements associated University A and B was reversed.

After reading the diversity frame statements, participants were asked to answer three questions. The first two questions asked about their preference toward University A and B: "How much do you like each school?" and "How much do you want to attend each school?" Then they were asked to rate how much their unfair experience affected their decision while choosing between University A and B. These questions in section 2 are designed to explore their preference for the different diversity frame which is the dependent variable in the mediation model.

In Section 3 the participants were asked to imagine their life after entering these two universities and to answer the following questions related to several aspects of their expectations for each school. The compiling of the questions in this section is based on the three mediators in our model: "value", "belonging" and "being treated with bias".

Participants provided ratings for each school separately. First, to measure "value", they were asked "How much do you think you can benefit from entering these universities?", for measuring sense of "belonging", the participants were asked "To what extent do you think you would feel more accepted in?", and for measuring "being treatment with bias", they were asked "How much do you think you may be treated with bias in these two universities?". The last questions they 
were asked was about the degree to which they expected to feel like part of the minority versus majority independently for two universities on Likert scales with 1 as "Minority" and 7 as "Majority".

\section{Results}

In the first part of our results, we examine the relationship between our independent variable (i.e. schooling experience) and dependent variable (i.e. attending preference for different universities with diversity frames). As we did not find a significant relationship between IV and DV, we further examined the relationship between schooling experience and perceived minority/majority, and the relationship between schooling experience and unfair/biased experience. The analyzing method used in this section is one-way ANOVA.

\subsection{Schooling Experience and Attending Preference}

To examine whether experience of studying abroad influenced the attending preference of high school students for imagined universities with either instrumental diversity frame or moral diversity frame, a one-way ANOVA, with abroad experience (never abroad vs. short term abroad vs. long term abroad) as a between-subjects factor, was conducted. Contrary to expectations, the ANOVA did not reveal a significant main effect of schooling experience abroad, $F(2,99)=$ $0.551, p=0.578$, indicating that participants with different schooling experience, overall, did not have significant difference on diversity frame-never abroad ( $M$ $=4.22, S D=1.553)$, short-term abroad $(M=4.60, S D=1.384)$, long-term abroad $(M=4.27, S D=1.614)$ (see Figure 2).

\subsection{Schooling Experience and Perceived Minority/Majority, Unfair/Biased Experience}

Then we want to examine whether the abroad schooling experience of Chinese high school students influenced their perception of themselves as minority or majority, a one-way ANOVA, with abroad experience (never abroad vs. short-term abroad vs. long term abroad) as a between-subjects factor, was conducted. Contrary

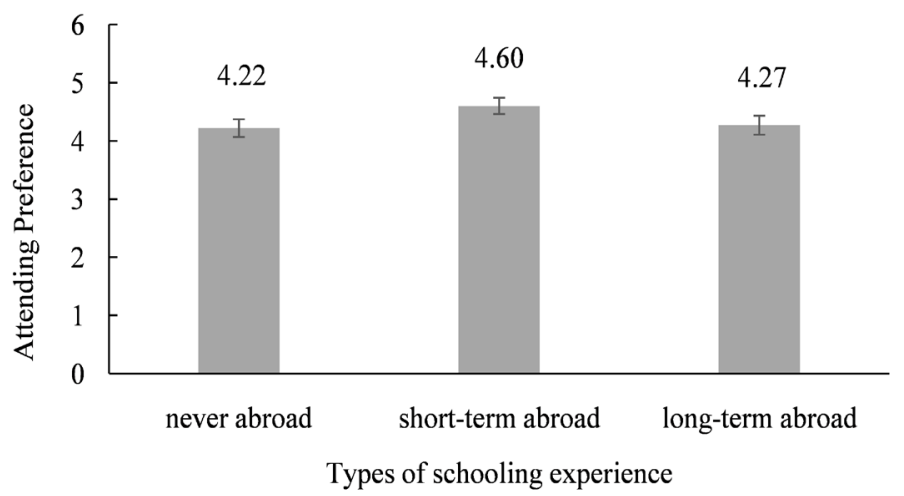

Figure 2. Attending preferences for students with different types of schooling experience. 
to expectations, the ANOVA did not reveal a significant main effect of abroad experience, $F(2,99)=0.891, p=0.413$, such that participants with different abroad experience, overall, did not have significant difference on their perception of themselves as minority or majority-never abroad $(M=4.51, S D=1.528)$, short-term abroad $(M=4.72, S D=1.792)$, long-term abroad $(M=4.12, S D=1.774)$ (see Figure 3). We also examined whether the abroad schooling experience of Chinese high school students influenced the severity degree of their unfair/biased experience, a one-way ANOVA, with abroad experience (never abroad vs. short term abroad vs. long term abroad) as a between-subjects factor, was conducted. For those who reported that they had never been treated unfairly or with bias based on nationality, we transformed their severity index into 0 when doing the analysis.

Contrary to expectations, the ANOVA did not reveal a significant main effect of abroad experience, $F(2,99)=1.423, p=0.246$. Even though we do not have the significant main effect, we still want to see the simple effect. The result showed that we have marginal significant simple effect that Chinese high school students who have never studied abroad $(M=0.92, S D=1.927)$ had lower severity of unfair/biased experience, and students who have short-term abroad experience $(M=1.76, S D=2.185)$ had higher severity of unfair/biased experience, $p$ $=0.108$ (see Figure 4$)$.

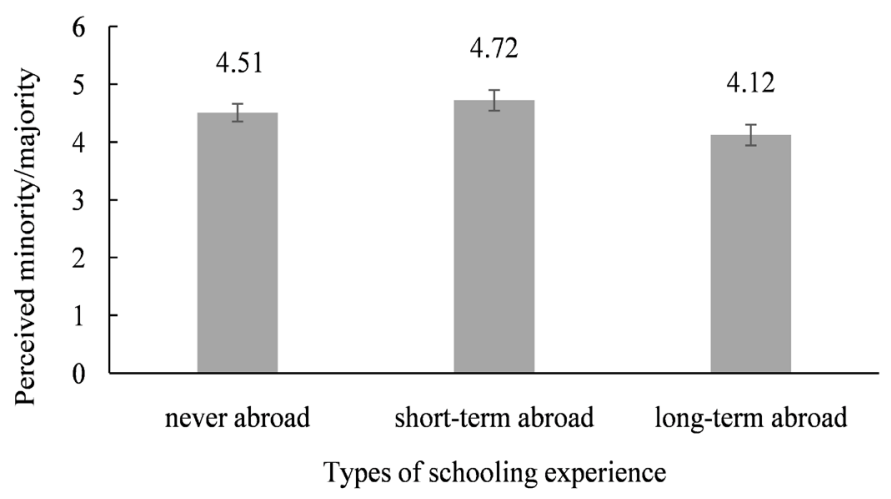

Figure 3. Perceived minority/majority for students with different types of schooling experience.

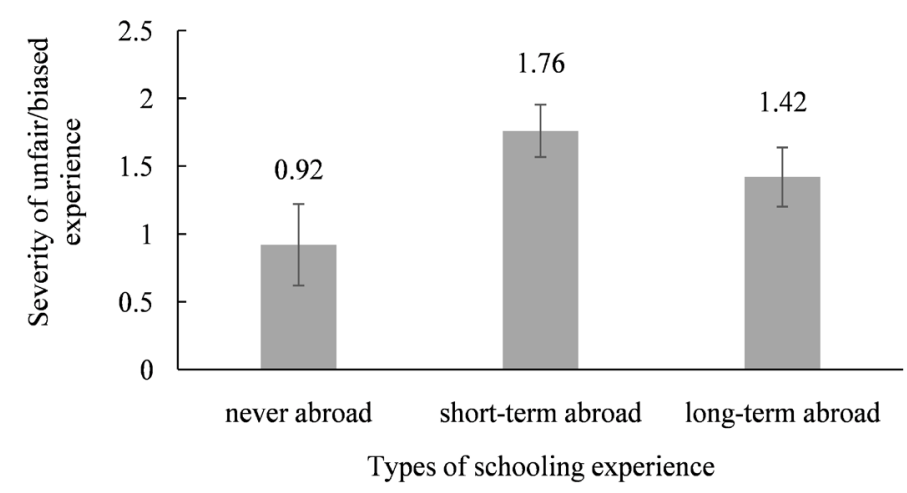

Figure 4. Severity of unfair/biased experience perceived by students with different types of schooling experience. 


\subsection{Perceived Minority/Majority, Unfair/Biased Experience and Attending Preference}

Because the above results showed that the hypothesized relationship between abroad experience and attending preference, the abroad experience and perceived minority/majority, the abroad experience and degree of severity of unfair/biased experience are not significant. In this end, we wanted to explore the correlation between perceived minority/majority and their attending preference, and correlation between unfair/biased experience and their attending preference.

In the next two parts, we focused on participants' perceived minority/majority and tested its relationship with the dependent variable (i.e. attending preference) and three mediators (i.e. expected value, sense of belonging and possibility of being treated with bias). The analyzing method used in this section is correlation.

\subsection{Perceived Minority/Majority and Attending Preference}

To test whether participants' perceived minority/majority could lead to different preference of diversity frames, we first tested the correlation between participants' perceived minority/majority and their attending preference for each school, and we got a marginal negative correlation $(r=-0.154, p=0.123)$.

\subsection{Perceived Minority/Majority and Expected Value, Sense of Belonging and Possibility of Being Treated with Bias}

Then we tested the correlation between participants' minority/majority and their expectations of instrumentally and morally motivated universities. The same as what we had expected, a significant positive correlation was found between participants' perceived minority/majority and their expectations for sense of belonging at the instrumentally motivated university $(r=0.325, p=0.001)$. A marginal significant negative correlation was found between participants' perceived minority/majority and their expected possibility of being treated with bias in the instrumentally motivated university $(r=-0.168, p=0.091)$. Correlations between perceived minority/majority and other variables were not significant (see Table 1).

\subsection{Severity of Unfair/Biased Experience Based on Nationality and Attending Preference}

In this part, we analyzed the correlation between participants' perceived severity of their unfair/biased experience based on nationality and their attending preference for each university in order to test whether unfair/biased experience could lead to different preference of different diversity frames. The result demonstrated that there was no significant correlation between these two variables $(r=0.103, p$ $=0.305)$. So, we didn't do further analysis as severity of unfair/biased experience couldn't lead to participants' preferences of different diversity frames as a predictor. 
Table 1. Pearson correlations between perceived minority/majority status and other variables (I: instrumental, M: moral).

\begin{tabular}{|c|c|c|c|c|c|c|c|c|}
\hline Measure & 1 & 2 & 3 & 4 & 5 & 6 & 7 & 8 \\
\hline $\begin{array}{l}\text { 1. Perceived } \\
\text { minority/majority }\end{array}$ & - & - & - & - & - & - & - & - \\
\hline $\begin{array}{l}\text { 2. Attending } \\
\text { preference }\end{array}$ & -0.154 & - & - & - & - & - & - & - \\
\hline 3. I-value & 0.145 & $-0.360^{\star * *}$ & - & - & - & - & - & - \\
\hline 4. M-value & -0.106 & $0.494^{* * *}$ & 0.002 & - & - & - & - & - \\
\hline 5. I-belonging & $0.325^{* *}$ & $-0.330^{\star *}$ & $0.553^{* * *}$ & 0.099 & - & - & - & - \\
\hline 6. M-belonging & 0.103 & $0.356^{* * *}$ & 0.135 & $0.499^{* * *}$ & $0.212^{\star}$ & - & - & - \\
\hline 7. I-bias & -0.168 & $0.241^{*}$ & -0.124 & 0.100 & $-0.369^{* * *}$ & 0.104 & - & - \\
\hline 8. M-bias & 0.134 & $-0.209^{*}$ & -0.133 & -0.192 & -0.040 & $-0.270^{\star *}$ & $0.313^{* *}$ & - \\
\hline
\end{tabular}

${ }^{\star} p<0.05,{ }^{* *} p<0.01,{ }^{* * *} p<0.001$.

This last part focused on the relationship between three mediators (i.e. expected value, sense of belonging and possibility of being treated with bias) and the dependent variable (i.e. attending preference). The analyzing method used in this section is linear regression.

\subsection{Expected Value, Sense of Belonging, Possibility of Being Treated with Bias, and Attending Preference}

At last, we conducted a linear regression analysis with the expected value, sense of belonging, and possibility of being treated with bias rated on 7-point Likert scales by high school students respectively for University A and B as predictors of the attending preference for university with different diversity frames. There was no covariate in the regression we conducted.

Expected value was significantly related to attending preference negatively at the university associated with the instrumental diversity frame $(\beta=-0.273, p=$ 0.003 ) and positively at the university associated with the moral diversity frame $(\beta=0.375, p=0.000)$.

Similarly, participants' ratings for sense of belonging based their imagined life were also significantly related to attending preferences for universities, notably, the sense of belonging was negatively related in instrumentally motivated university $(\beta=-0.220, p=0.032)$ and positively related in morally motivated universities $(\beta=0.194, p=0.035)$. Ratings for expected possibility of being treated with bias after entering university associated with instrumental diversity frame weren't related to attending preference $(\beta=-0.120, p=0.182)$. However, ratings for expected possibility of being treated with bias were marginally related to morally motivated university $(\beta=-0.167, p=0.053)$. See Table 2 for linear regression analysis predicting attending preference for instrumentally \& morally motivated universities. 
Table 2. Linear regression analysis predicting attending preference for instrumentally \& morally motivated universities.

\begin{tabular}{ccccc}
\hline & $\beta$ & S.E. & $t$ & $p$ \\
\hline Instrumentally motivated university & & & & \\
Expected value & -0.273 & 0.120 & -3.012 & 0.003 \\
Sense of belonging & -0.220 & 0.119 & -2.179 & 0.032 \\
Possibility of being treated with bias & 0.120 & 0.102 & 1.345 & 0.182 \\
$\quad \begin{array}{l}\text { Morally motivated university } \\
\text { Expected value }\end{array}$ & 0.375 & 0.114 & 4.379 & 0.000 \\
$\quad$ Sense of belonging & 0.194 & 0.120 & 2.144 & 0.035 \\
Possibility of being treated with bias & -0.167 & -0.169 & -1.960 & 0.053 \\
\hline
\end{tabular}

\section{Discussion}

The goal of this study was to explore the relationship between Chinese high school students' schooling abroad experience and their preference of instrumental or moral diversity frame, and further study the mediation mechanism. However, the statistical results were partly contrary to our expectations.

Our first hypothesis about schooling abroad experience (i.e. the independent variable) and the preference of different diversity frames (i.e. the dependent variable) was rejected as the main effect of schooling experience on the preference of diversity frame was not significant, which means that whether someone has studied abroad or not won't influence their preference for diversity frames. Our second hypothesis about the relationship between schooling experience and two mediators (i.e. severity of unfair/biased experience and perceived minority/majority) was partly accepted, as the only significant difference was between the severity of unfair/biased experience of students who had never studied abroad and those who only had short-term schooling abroad experience. This means that Chinese high school students who had short-term schooling abroad experience did experience more severe bias based on nationality compared with those who had never studied abroad, while schooling abroad experience can't predict someone's perception of his or her minority/majority status.

Though these results don't match with our hypotheses, there are several reasons that can help explain them. The first possible reason is that Chinese high school students are Asians, and Asians' status in America is somewhat different from other racial minorities' (i.e. Black Americans or LatinXs), so that their preference of diversity frames could be different as well. Starck, Sinclair and Shelton (2019a) studied Asian parents' preference while choosing university for their children, and found out that even preference for the morally motivated university is pronounced among African American participants and LatinX participants, Asian participants showed no preference between the two. This may be due to the fact that Asian are least considered as underrepresented groups in higher education settings. According to The Higher Education Act in the USA, the term 
"minority" only refers to American Indian, Alaskan Native, Black, Hispanic, Pacific Islander, or other ethnic group underrepresented in science and engineering. Though it is also noted that identification of a particular group as underrepresented may vary by discipline, which means that Asian Americans in social sciences and humanities fields are also minorities, it's still clear that Asians are evidently considered differently compared with other minor races in America. The reasons behind may relate to those stereotypes which Americans have toward Asians. Fiske et al. (2002) found that Asians are most often considered to be highly competent but cold, which are different from other minority groups which are considered to be weaker and lower competent, thus other minor races may elicit less threat than Asians. As a result, not only does The Higher Education Act not define Asian Americans as minority, but it is also common that other Americans' attitudes towards Asians are different from those towards other racial minorities.

Though the relationship between schooling abroad experience was not significant, our test results of the correlation between perceived minority/majority, severity of unfair/biased experience and the preference of diversity frames showed that the more someone perceived him or herself as majority, the more he or she preferred the instrumentally motivated university, which matched with our expectations. However, the severity of unfair/biased experience had no significant correlation with preference of diversity frames. This could be due to the subjectivity of the rating and individual differences when considering the importance of unfairness when choosing universities. We split the data based on their length of schooling abroad experience to see for which group of people that their severity of unfair experience may influence their preference for diversity frames. The results showed that only for those who had short-term abroad that the severity of unfair experience had a significant positive correlation with their attending preference for each university $(r=0.422, p=0.036)$, which meant that the more severe their unfair experience was, the more they preferred to attend the morally motivated university. It didn't quite match our hypothesis that the longer someone's schooling abroad experience was, the more he or she preferred the morally motivated university. Nevertheless, this could be partly explained by the process of adjustment or integration. For Chinese high school students who chose to study abroad for some time, the longer they stayed abroad, the more opportunities they would have to join new groups, and being friends with surrounding people. And these kinds of connections could generally make a positive contribution to their well-being, especially in the context of adjusting to life transitions (Ng, Haslam, Hslam, \& Cruwys, 2018). Also, Trueba (2004) found that immigrants with a longer length of stay in the USA showed more tendency towards adjustment attitude whereas those who resided for a shorter period of time demonstrated more acculturative stress. As a result, those who have longer schooling abroad experience may be more adapted to the culture they are in, and then encounter or at least they perceive themselves encountering less unfairness in daily life. 
The test of correlation between perceived minority/majority and participants' expectations of the value, sense of belonging and possibility of being treated with bias in morally and instrumentally motivated universities demonstrated that perceived minority/majority only influenced participants' expected sense of belonging and possibility of being treated with bias in the instrumentally motivated university but not in the morally motivated university. This suggested that everyone expected themselves to be more accepted and treated with less bias in the morally motivated university. This matched Starck et al. (2019a)'s results that racial minority parents' believed that their kids would belong more and experience less bias in the morally motivated university. Considering Chinese high school students were all minorities, it's reasonable for us to get such results about their expectations in the morally motivated university.

Finally, the regression analysis showed that three mediators (i.e. value, sense of belonging, possibility of being treated with bias) were all significant predictors for their preference of different diversity frame, except for the expected possibility of being treated bias at the instrumentally motivated university. This could also be explained by different people considering bias as different importance when choosing universities, as after we controlled "the extent to which their unfair experience affected their decision while choosing between universities", the correlations between their expected possibility of being treated with bias and attending preference for two universities were significant $(p<0.05)$ (i.e. the more bias they expected to experience, the less they prefer the university).

\section{Conclusion}

Previous literatures seldom focus on the rationale behind promoting diversity, and the concept of diversity frame has been little studied as well, especially in places other than the United States. In the current study, we focus on Chinese high school students and we have expected that those who have schooling abroad experience tend to present a stronger preference toward moral diversity frame. Though our final results are partly contrary to the expectations-we didn't find a significant relationship between schooling abroad experience and preference of different diversity frames, they proved the potential influence of factors like perceived minority/majority which is much more subjective and nuanced compared to those more frequently studied variables like race or other environmental factors.

\section{Limitations and Future Directions}

There are several limitations to this study. First, our sample size is somewhat small after splitting the participants into three groups based on their length of schooling abroad experience, as there were only 25 participants for short-term and 26 participants for long-term group. In this case, the analysis based on schooling abroad experience might be less stable and more difficult to get significant results. Second, our sample might be biased as we only delivered questionnaires face-to-face asking them to fill our questionnaire without paying them remuneration. As there were many groups sending out questionnaires during 
those days, those who agreed to fill our questionnaire could be more friendly and cared more about inclusion and harmony than those who refused. In this case, people who were potential to prefer instrumental diversity frame might not be included in our data. To improve the representativeness of our participants, we could either attach adequate reward (i.e. $\$ 0.5$ (Trawalter et al., 2015)) to our questionnaire or cooperate with high school teachers and ask all the students in class to fill out the questionnaire. Another limitation of this study is the index we choose to measure our unfair/biased experience. As mentioned above, the degree of the severity might be too subjective and without reliable standards, so that we could choose to modify the measurement of unfair/biased experience into a combination of frequency and severity in future studies.

One implication of our study is studying the preference of Chinese high school students' preference of different diversity frames, possible influencing factors and mediators. Our findings suggest that different from diversity frame studies based on racial majorities or minorities in the United States, the case of Chinese high school students is more complex and nuanced, so apart from more environmental and extrinsic variables like schooling abroad experience and unfair/biased experience, those more intrinsic and stable variables like perceived minority/majority or personality traits need more attention. Another implication of our study is we proved that Chinese high school students tend to possess stable positive expectations for the morally motivated university no matter his or her backgrounds. This suggests that every university could add morally motivated statement to their websites about diversity and inclusion to attract more racial minority students.

Based on further analysis of our data, we come up with several directions for future research. The first one is about gender differences: we've found out in our current study that male students expected higher sense of belonging in instrumentally motivated school compared with female students, and female students expected themselves to belong to majority in morally motivated school more than male students did. Therefore, future research could further explore why these differences exist and whether gender could be a moderator between schooling abroad experience and the preference of diversity frames. Additionally, future research could focus on the influence of school location. Previous studies about diversity frames are all conducted in American settings, while participants' school locations were in different countries (e.g. Singapore, Switzerland etc.) in the present study. Considering the racial issues and emphasis of diversity vary in different countries, it could be better to look at the influence of schooling abroad experience separately for different countries and then compare those results.

\section{Acknowledgements}

The research study was conducted under the guidance of Professor Stacey Sinclair from Princeton University during CIS program in Shanghai. The authors would like to thank Professor Stacey Sinclair, Hermione Wang and other class- 
mates in the same program for their significant suggestions on our research and the drafts of our paper.

\section{Conflicts of Interest}

The authors declare no conflicts of interest regarding the publication of this paper.

\section{References}

Fiske, S. T., Cuddy, A. J. C., Glick, P., \& Xu, J. (2002). A Model of (Often Mixed) Stereotype Content: Competence and Warmth Respectively Follow from Perceived Status and Competition. Journal of Personality \& Social Psychology, 82, 878-902. https://doi.org/10.1037/0022-3514.82.6.878

Heng, T. T. (2018). Different Is Not Deficient: Contradicting Stereotypes of Chinese International Students in US Higher Education. Studies in Higher Education, 43, 22-36. https://doi.org/10.1080/03075079.2016.1152466

Hoxworth, L. (2018). Understanding the Benefits of School Diversity in the Majority-Minority Age.

https://news.virginia.edu/content/understanding-benefits-school-diversity-majority-mi nority-age

Nabi, Z. (2016). Studying Abroad: Pros and Cons. https://www.topuniversities.com/blog/studying-abroad-pros-cons

Ng, N. W. K., Haslam, S. A., Haslam, C., \& Cruwys, T. (2018). "How Can You Make Friends If You Don't Know Who You Are?” A Qualitative Examination of International Students' Experience Informed by the Social Identity Model of Identity Change. Journal of Community \& Applied Social Psychology, 28, 169-187.

https://doi.org/10.1002/casp.2349

Starck, J. G., \& Sinclair, S. (2019). Identity- and Ideology-Based Moderators of Diversity Rationales [Unpublished Manuscript]. Princeton, NJ: Princeton University.

Starck, J. G., Sinclair, S., \& Shelton, N. S. (2019a). The Case for Diversity: A Matter of Value or Values? [Unpublished Manuscript]. Princeton, NJ: Princeton University.

Starck, J. G., Sinclair, S., \& Shelton, N. S. (2019b). The Costs of Emphasizing Value: How Instrumental Diversity Rationales Compromise the Promise of Racial Equity [Unpublished Manuscript]. Princeton, NJ: Princeton University.

Trawalter, S., Driskell, S., \& Davidson, M. N. (2015). What Is Good Isn't Always Fair: On the Unintended Effects of Framing Diversity as Good. Analyses of Social Issues and Public Policy, 16, 69-99. https://doi.org/10.1111/asap.12103

Trueba, E. T. (2004). The New Americans: Immigrants and Transnational at Work. Lanham, MD: Rowman \& Littlefield Publishers.

Vorauer, J. D., Hunter, A. J., Main, K. J., \& Roy, S. A. (2000). Meta-Stereotype Activation: Evidence from Indirect Measures for Specific Evaluative Concerns Experienced by Members of Dominant Groups in Intergroup Interaction. Journal of Personality and Social Psychology, 78, 690-707. https://doi.org/10.1037/0022-3514.78.4.690

Wong, Y. J., Tsai, P. C., Liu, T., Zhu, Q., \& Wei, M. (2014). Male Asian International Students' Perceived Racial Discrimination, Masculine Identity, and Subjective Masculinity Stress: A Moderated Mediation Model. Journal of Counseling Psychology, 61, 560. https://doi.org/10.1037/cou0000038 